\title{
High-resolution femtosecond pulse shaping
}

\author{
A. M. Weiner, J. P. Heritage, and E. M. Kirschner \\ Bell Communications Research, 331 Newman Springs Road, Red Bank, New Jersey 07701-7020
}

Received February 8, 1988; accepted April 25, 1988

\begin{abstract}
The synthesis of arbitrarily shaped femtosecond pulses by spectral filtering in a temporally nondispersive grating apparatus is demonstrated. Spectral filtering is accomplished by utilizing spatially patterned masks to modify the amplitude and the phase of the optical frequency components that are spatially dispersed within the apparatus. We are able to pattern spectra over a large dynamic range (approaching $10^{4}$ ) and with unprecedented resolution. We illustrate the power of this technique by synthesizing a number of femtosecond waveforms, including femtosecond tone bursts with terahertz repetition rates, picosecond square pulses with 100 -fsec rise times, and highly complex pseudonoise bursts produced by spectral phase encoding.
\end{abstract}

\section{INTRODUCTION}

Shaped, modulated, and encoded waveforms are utilized for a variety of applications in communications, remote sensing, signal processing, and spectroscopy. In spread spectrum communications, for example, system capabilities such as interference rejection, error correction, and multiplexing are achieved by means of specially coded signal formats. ${ }^{1}$ A means for generating appropriately shaped waveforms is crucial for any of these applications.

In the context of ultrafast optics a number of researchers have used Fourier synthesis techniques to generate specially shaped ultrashort pulses. In the 1970's, Desbois et al. ${ }^{2}$ and Agostinelli et al. ${ }^{3}$ demonstrated a technique for optical pulse shaping based on spatial filtering of optical frequency components that were physically dispersed by diffraction gratings. Because of the temporal dispersion of the gratings, however, the shaped pulses were chirped, and even their shortest features were stretched in time compared with the input pulse. More recently Froehly et al. demonstrated true Fourier-transform pulse shaping on a 30-psec time scale by utilizing a grating apparatus free from temporal dispersion; ${ }^{4}$ others ${ }^{5-8}$ produced shaped picosecond pulses shorter than the input pulse by spatial filtering within a fiber and grating pulse compressor. Picosecond pulse shaping by temporal modulation and subsequent compression of chirped optical pulses has also been reported. ${ }^{9}$

In this paper we demonstrate, for the first time to our knowledge, the synthesis of arbitrarily shaped femtosecond pulses. Previously we shaped picosecond pulses by spatial filtering within a fiber and grating pulse compressor ${ }^{5-8}$; here, we shape femtosecond pulses from a colliding-pulse modelocked (CPM) laser ${ }^{10,11}$ by spatial filtering in a nondispersive grating apparatus. Both amplitude and phase can be controlled, and the synthesized waveforms can be simple or highly complicated. The stability and the bandwidth of our system make it possible to pattern spectra over a large dynamic range (approaching 104) and with unprecedented resolution. We illustrate this powerful technique by synthesizing a number of femtosecond time-scale waveforms, including square pulses, terahertz-repetition-rate tone bursts, ${ }^{12}$ and highly complex pseudonoise bursts. ${ }^{13}$ Possible applica- tions for these unique femtosecond waveforms are indicated throughout this paper.

The paper is structured as follows. We describe the apparatus used for our femtosecond pulse-shaping experiments in Section 2, and in Section 3 we present several examples of unusually shaped pulses that were generated by particularly simple spectral masks. Waveforms that require detailed and precise spectral control and that challenge the capabilities of our technique are reported in Section 4 . Constraints that govern the application of our technique are enumerated in Section 5. In Section 6 we present new results on encoding and decoding of femtosecond pulses, which we previously proposed as a potential basis for ultrashort pulse codedivision multiple-access communications. ${ }^{12,13}$ In Section 7 we summarize.

\section{EXPERIMENTAL APPARATUS}

The experimental arrangement consists of a femtosecond laser, a pulse-shaping grating apparatus, and diagnostics for measuring the intensity profiles and the spectra of the shaped pulses. As a source of femtosecond pulses, we utilize a dispersion-compensated, CPM ring dye laser, ${ }^{10,11}$ which provides typical pulse durations of $75 \mathrm{fsec}$ at a wavelength of $0.62 \mu \mathrm{m}$. The pulse-shaping apparatus, shown schematically in Fig. 1, consists of a pair of 1700 -line/mm gratings placed at the focal planes of a unit magnification confocal lens pair. ${ }^{4}$ The grating separation is $60 \mathrm{~cm}$, and the lenses are achromatric with focal lengths of $15 \mathrm{~cm}$. Spatially patterned amplitude and phase masks are inserted midway between the two lenses at the point where the optical spectral components experience maximal spatial separation. The pulse shape at the output of the apparatus is the Fourier transform of the pattern transferred by the masks onto the frequency spectrum. Intensity profiles of the shaped pulses are measured by cross correlation using femtosecond pulses directly out of the CPM laser as the reference; the corresponding spectral profiles are measured using a $0.32-\mathrm{m}$ spectrometer and a linear photodiode array.

The grating setup shown in Fig. 1 is to be contrasted with the Treacy grating pair, ${ }^{14,15}$ which is commonly used for fiber 

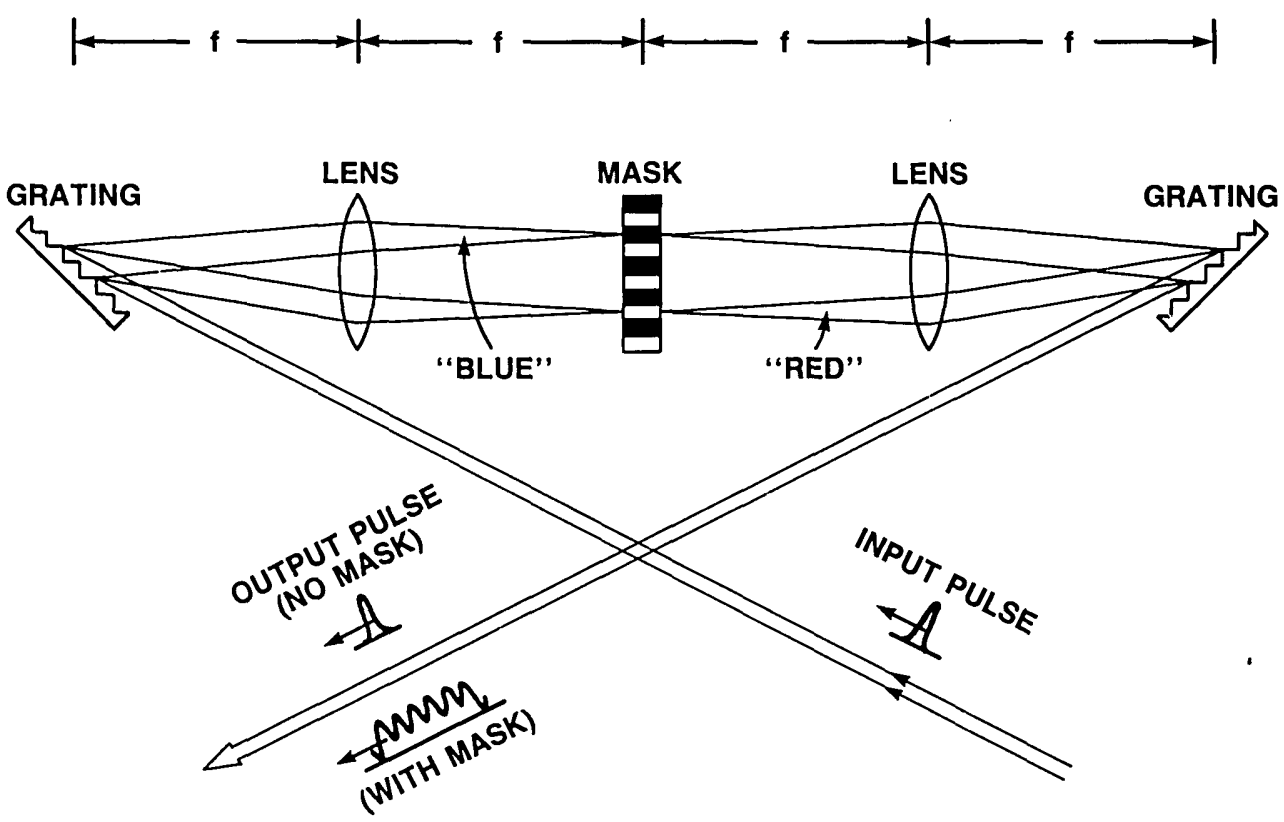

Fig. 1. Dispersion-free pulse-shaping apparatus.

and grating pulse compression and which we used for our previous demonstrations of pulse shaping. ${ }^{5-7}$ In those experiments chirped pulses emerging from an optical fiber are compressed because of the negative group-velocity dispersion of the Treacy grating pair; the bandwidth-limited compressed pulses that result are then suitable for Fouriertransform pulse shaping. Because pulses from the CPM laser are substantially bandwidth limited, in this paper we employ the dispersion-free configuration sketched in Fig. 1. This geometry was utilized previously by Froehly et al. for pulse shaping with 30-psec Nd:YAG laser pulses. ${ }^{4}$ Our experiments, using pulse durations as much as 600 times shorter than in Ref. 4, test the dispersion-free character of the apparatus much more stringently.

We have verified that our pulse shaper is dispersion free even for input pulses as short as 48 fsec. Figure 2 compares the autocorrelation trace of 48 -fsec pulses directly from the CPM laser with the autocorrelation of pulses that have emerged from the shaper (with no mask present). The pulse widths are identical. For comparison, a Treacy grating pair with the same $60-\mathrm{cm}$ grating separation would broaden these pulses to approximately 55 psec.

As an aside, we note that a generalized grating compressor with either positive or negative dispersion may be obtained by moving the gratings either closer to or farther from the lenses, respectively. Thus the reduced wings evident in the output pulse of Fig. 2 may be attributed to cancellation of some small chirp on the pulses from the CPM laser. The generalized grating compressor has been studied theoretically by Martinez. ${ }^{16}$ Experimentally we have used such a compressor in the negative-dispersion regime to achieve subpicosecond compression and high-resolution shaping of 1.06- $\mu \mathrm{m} \mathrm{Nd:YAG} \mathrm{laser} \mathrm{pulses.}{ }^{17}$ A similar compressor operating in the positive-dispersion regime was demonstrated in Ref. 18.

Spatially patterned masks are a key component of our pulse-shaping experiments, and some comments concerning their design and fabrication are in order. For many experiments we utilize two masks, one to control the spectral phases and the second to control the amplitudes. The phase masks that we used are binary; with the phase at each point on the mask set to either zero or $\pi$. The masks are fabricated on fused-silica substrates; the $\pi$-phase retardations are obtained by reactive-ion etching to a depth $D=\lambda / 2(n-1) \simeq$ $0.68 \mu \mathrm{m}$, where $n$ is the refractive index of the fused silica. Amplitude masks consist of opaque titanium-gold films evaporated onto the fused silica. For the phase masks and for binary amplitude masks (in which the transmission is either 0 or 1), patterning is achieved by standard microlithography. Because the optical spectrum is spread into a linear, fan-shaped beam, patterning is required in only one dimension. The second dimension is used to fit several patterns onto each substrate. For some waveforms, such as the square pulse, the amplitude mask must provide a continuously variable transmission function. For such masks we use a special fabrication technique, which we describe later in this paper.

The connection between the physical mask and the spec-

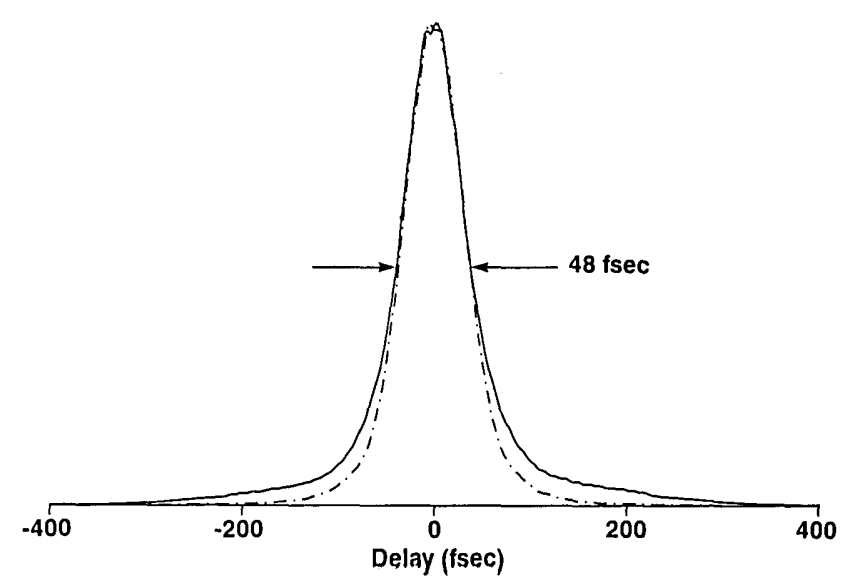

Fig. 2. Autocorrelation traces of 48-fsec pulses from the CPM laser, measured before (solid curve) and after (dashed-dotted curve) the grating apparatus. With no mask present, the pulse widths are identical. 
tral filter that results is discussed in Ref. 8. The linear dimension of the patterns is determined by the spatial dispersion of the optical beam, given by $\delta \lambda / \delta x=\left(d \cos \theta_{d}\right) / f$, where $f$ is the focal length, $d$ is the grating period, and $\theta_{d}$ is the diffraction angle. For $f=15 \mathrm{~cm}, 1 / d=1700 \mathrm{~mm}^{-1}$, and $\theta_{d}=35^{\circ}$, we compute $\delta \lambda / \delta x$ to be $3.22 \mathrm{~nm} / \mathrm{mm}$, in reasonable agreement with the measured value of $3.37 \mathrm{~nm} / \mathrm{mm}$. To accommodate the entire CPM-laser bandwidth $(\simeq 10-\mathrm{nm}$ FWHM), an aperture of approximately $5 \mathrm{~mm}$ is required. In many cases we work with $2.5-\mathrm{mm}$ apertures in order to justify the simplifying approximation that the input spectrum is flat over the entire spectral range.

\section{SIMPLE MASKS: SPECTRAL WINDOWING, TONE BURSTS, AND ODD PULSES}

As first examples of femtosecond pulse shaping, we demonstrate some simple masking operations that we previously demonstrated on the picosecond time scale. ${ }^{5,6}$ These examples are chosen to illustrate the fine control and the large dynamic range that our technique affords.

We first consider spectral windowing, which is the use of an amplitude mask consisting of a single slit in order to truncate the optical bandwidth. Spectral windowing was utilized previously within a fiber and grating pulse compressor to eliminate portions of the spectrum that were not linearly chirped and thus were unsuitable for pulse compression. ${ }^{5}$ It was utilized even earlier to perform slicing of longer chirped pulses that had been stretched by grating pairs. ${ }^{2,3}$ In the present case our pulses are already bandwidth limited; spectral windowing reduces the optical bandwidth and increases the pulse duration. As an example, we utilized a window to produce a rectangular frequency spectrum with a bandwidth of $1.5 \mathrm{~nm}$. The resulting pulse shape is the wellknown sinc function given by

$$
E(t)=E_{0} \frac{\sin (\pi \Delta f t)}{(\pi \Delta f t)}
$$

where $\Delta f$ is the bandwidth. The intensity profile of the windowed pulse, measured by cross correlation, is shown in Figs. 3(A) and 3(B) on a linear and a semilog scale, respectively. From Fig. 3(A), the duration of the sinc-function pulse is 790-fsec FWHM and the time-bandwidth product $\Delta f \Delta t$ is 0.92 , in good agreement with the predicted duration of $760 \mathrm{fsec}$ and the theoretical time-bandwidth product of 0.886 . The time interval between minima, evident from Fig. $3(\mathrm{~B})$, is $\simeq 849 \mathrm{fsec}$, also in very close agreement with the calculated value of $854 \mathrm{fsec}$. As evident from the semilog plot, the correct pulse shape is obtained for several orders of magnitude.

A simple extension is to use an amplitude mask that consists of two slits in order to select two isolated spectral components. The two frequencies will interfere, producing a high-frequency tone burst. A cross-correlation measurement of such a femtosecond tone burst is shown in Fig. 4(A) for a frequency separation of $3.37 \mathrm{~nm}(2.6 \mathrm{THz})$. The cross correlation shows some 20 distinct temporal peaks, with an estimated order-of-magnitude increase in complexity possible. The 2.6-THz beat frequency, corresponding to a period of only $380 \mathrm{fsec}$, is an order of magnitude faster than the highest-frequency tone bursts generated by other techniques. ${ }^{19}$ By introducing a phase mask in order to phase shift one frequency component by $\pi$, we can interchange the
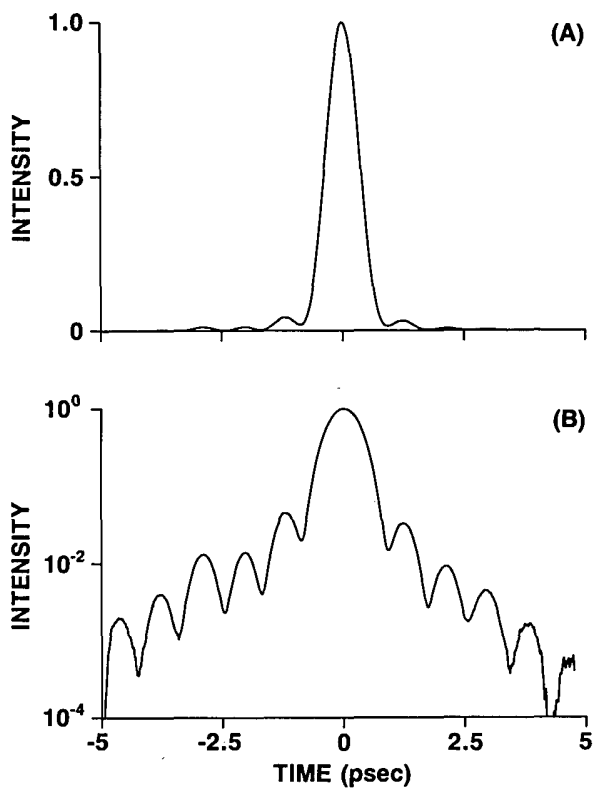

Fig. 3. Cross-correlation measurement of a sinc-function pulse: (A) linear plot, (B) semilog plot.
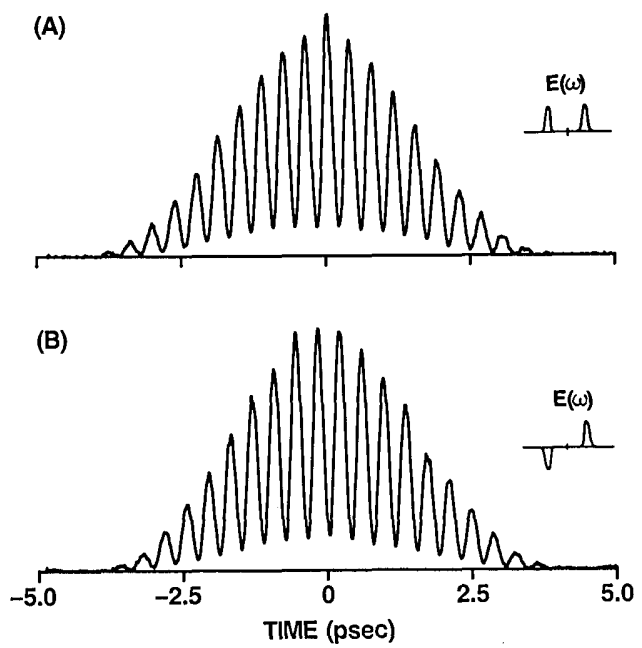

Fig. 4. (A) Cross-correlation measurement of a $2.6-\mathrm{THz}$ burst of femtosecond pulses. (B) Shifted burst of pulses that results when one of the frequencies is given a $\pi$ phase shift.

positions of the peaks and nulls [Fig. 4(B)]. Thus the phase of the temporal beat note is determined by the relative phase of the two selected frequency components; conversely, by measuring the phase of the beat note as a function of the offset between the two selected frequencies, one could map out the phase spectrum of femtosecond pulses. Such twoslit temporal interference measurements were previously performed to determine the optical phase spectrum of compressed picosecond pulses. ${ }^{20}$

As a simple example of phase masking alone, we consider the odd pulse. When half of an originally symmetric spectrum undergoes a $\pi$ phase shift, it becomes an antisymmetric function. The corresponding electric field will be an odd function of time; the intensity profile will consist of two peaks separated by a null at $t=0$, where the phase of the carrier jumps abruptly by $\pi$. Such odd pulses were generated previously in picosecond pulse-shaping experiments ${ }^{6}$ and 


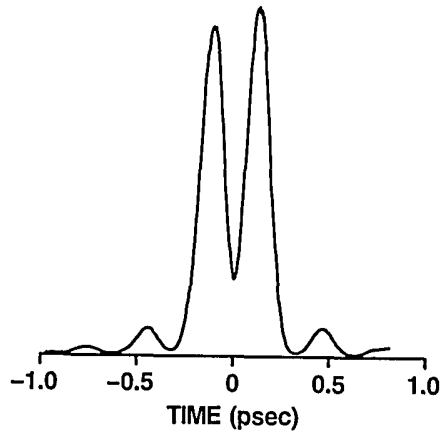

Fig. 5. Cross-correlation measurement of a femtosecond odd pulse.

are closely related to zero-area pulses produced by propagation through resonant atomic vapors. ${ }^{21}$ Figure 5 shows the cross-correlation measurement of a femtosecond odd pulse, generated using a total spectral width of $7.0 \mathrm{~nm}(5.5 \mathrm{THz})$. Except for the shorter time scale, this odd pulse is nearly identical with the picosecond odd pulse produced with the fiber and grating compressor. ${ }^{6}$ Femtosecond odd pulses and other related pulse shapes may prove useful for investigations of dark soliton ${ }^{22-24}$ pulse propagation in optical fibers.

\section{ADVANCED MASKS: PULSE TRAINS AND SQUARE PULSES}

The examples discussed above involve simple amplitude and phase masks. To generate more-general pulse shapes, more-detailed control of the spectrum will be required. In this section we discuss the application of sophisticated phase and amplitude masks for synthesizing, respectively, femtosecond pulse trains and optical square pulses.

\section{A. Pulse Trains}

The most familiar filter that could be used to generate a train of evenly spaced pulses would be an amplitude mask consisting of a set of evenly spaced slits on an opaque background. This approach was illustrated above in the discussion concerning Fig. 4. Although this approach does yield nice pulse trains, it suffers from poor energy efficiency. In this section we show how pure phase masks can be used to generate trains of femtosecond pulses with negligible loss. These phase masks, which can include $>150$ separate phase elements, provide a first example of highly detailed filtering operations. Additionally, the phase codes that we utilize for pulse-train generation will be revisited in Section 6 , in which we discuss a novel communication system based on encoding and decoding of ultrashort optical pulses.

An amplitude mask for pulse-train generation can be designed as follows:

$$
\left\{(100 \ldots .0)_{1}(100 \ldots 0)_{2} \ldots(100 \ldots 0)_{N / P}\right\} .
$$

The mask is divided into $N$ pixels, which are grouped into $N /$ $P$ periodic repetitions of the sequence $(100 . .0)$, which consists of a single 1 followed by $P-1$ zeros. 1 and 0 denote pixels at which the mask is transparent or opaque, respectively. Such a mask provides a train of roughly $P$ pulses within a smooth envelope but at the cost of throwing away a fraction $(P-1) / P$ of the incident energy.
To produce a similar pulse train by using phase alone, the mask should be designed as follows:

$$
\left\{\left(\Phi_{1} \Phi_{2} \Phi_{3} \ldots \Phi_{P}\right)_{1}\left(\Phi_{1} \Phi_{2} \Phi_{3} \ldots \Phi_{P}\right)_{2} \ldots\left(\Phi_{1} \Phi_{2} \Phi_{3} \ldots \Phi_{P}\right)_{N / P}\right\} \text {. }
$$

The mask is again divided into $N$ pixels grouped into $N / P$ periodic repetitions of a predetermined sequence of $P$ phases. In the notation above, $\left(\Phi_{1} \Phi_{2} \Phi_{3} \ldots \Phi_{p}\right)_{j}$ corresponds to the $j$ th repetition of the sequence and $\Phi_{i}$ denotes the $i$ th phase in the sequence. If each pixel is associated with a bandwidth $\delta f$, the total bandwidth is $N \delta f$, and the duration of the individual pulses in the train is of order $1 / N \delta f$. The period is $1 / P \delta f$, and the number of pulses in the train is of order $P$. A phase mask can be nearly lossless because the only loss mechanism is scattering from the edges of the individual pixels; if $N$ does not exceed the number of resolving spots (see Section 5), the loss will be small.

To produce a uniform or nearly uniform train of pulses, one must properly select the sequence $\left(\Phi_{1} \Phi_{2} \Phi_{3} \ldots \Phi_{p}\right)$. Suitable sequences are known and are widely used for designing antennas with broad radiation patterns and reflection phase gratings with broad diffraction patterns. These same sequences find numerous applications as codes for precision ranging and spread spectrum communications. In his book Number Theory in Science and Communication, ${ }^{25}$ Schroeder discusses a number of sequences that will be suitable for our use and enumerates a variety of fascinating applications.

We have fabricated phase masks based on repetitions of the so-called maximal length sequences ( $M$ sequences)..$^{1,25}$ The $M$ sequence, a binary-valued code of length $P=2^{L}-1$, where $L$ is an integer, is particularly well known in the context of spread spectrum communications. Each $\Phi_{i}$ in our mask is assigned a value of 0 or $\pi$, depending on whether the corresponding element of the $M$ sequence is a 0 or a 1 .

Illustrative pulse-shaping data are shown in Fig. 6(A) for a phase mask consisting of five repetitions of an $M$ sequence of length 15. For comparison, pulse trains generated using the corresponding amplitude mask are shown in Fig. 6(B); note the difference in the intensity scale! The details of the two pulse trains are identical except for the missing pulse at $t=0$ in the upper trace. Additional cross-correlation data, plotted in Figs. 6(C) and 6(D) on an expanded horizontal scale, demonstrate the similarity even more clearly. For these data the spectral window is $2.5 \mathrm{~mm}(N \delta f=6.575 \mathrm{THz}, \delta f=$ $0.0877 \mathrm{THz}$ ), yielding a pulse separation of $817 \mathrm{fsec}$ and a 150 -fsec pulse width. When the same phase mask is stretched to fit within a $5-\mathrm{mm}$ window $(\delta f=0.175 \mathrm{THz})$, the separation and the pulse width are reduced to 407 and 77 fsec, respectively [see Fig. 6(E)].

At this point several comments are in order. First, although pulse trains produced by phase masks and by amplitude masks exhibit similar intensity profiles, the phase profiles are quite different. For the amplitude masks, the phase of each pulse is identical, but for the phase masks, the phase varies from pulse to pulse in a manner determined by the particular code. Second, we note that the missing pulses at $t=0$ [in Figs. 6(A), 6(C), and 6(E)] are a property of the $M$-sequence code itself. Pulse trains with no pulses missing can be synthesized by using quadratic residue codes, ${ }^{25}$ for example, in place of $M$ sequences; and other codes could beutilized to produce specially tailored series of pulses. Tailored pulse trains will be useful for repetitive excitation of coherent molecular vibrations by impulsive stimulated $\mathrm{Ra}$ - 

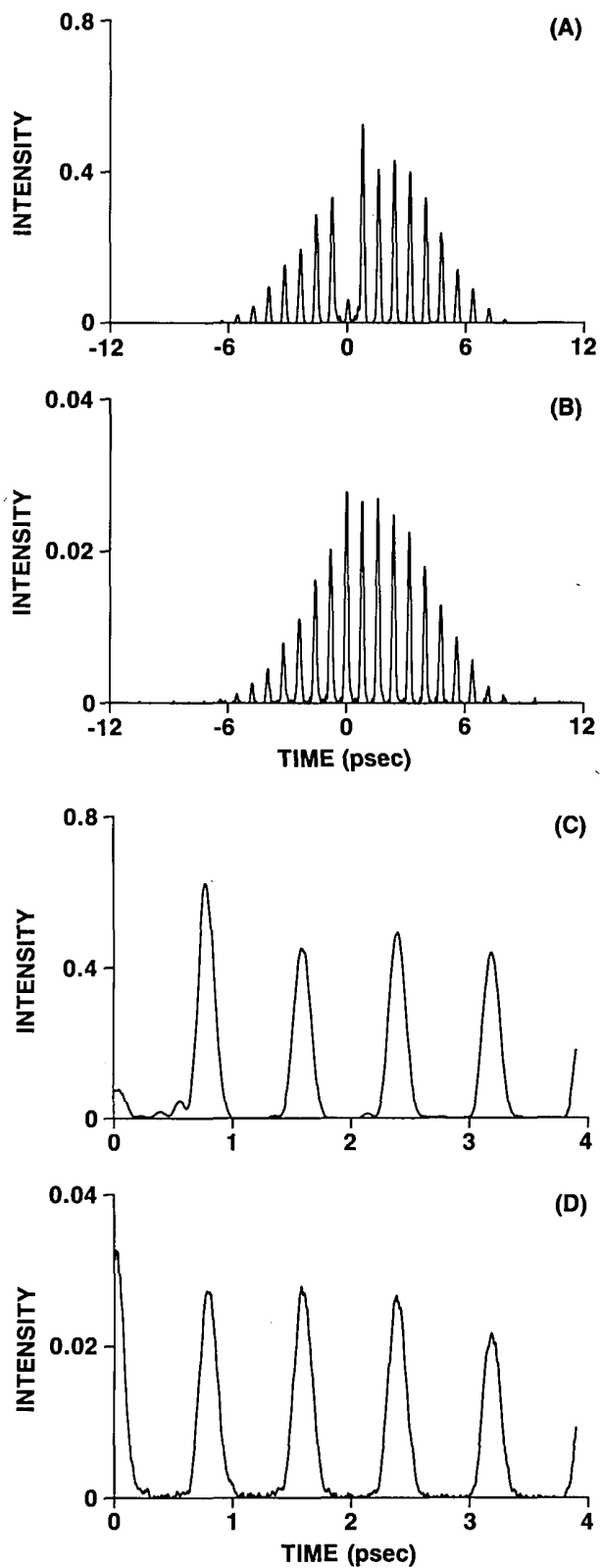

man scattering, ${ }^{26}$ in order to achieve mode selectivity and to excite large vibrational amplitudes. ${ }^{27}$

Finally, we note that the envelopes of the recorded waveforms are asymmetric about $t=0$ for amplitude masks as well as for phase masks. This asymmetry was unexpected and is not completely understood. The maximum temporal envelope of a shaped waveform depends on the width and the shape of the narrowest spectral feature, which in turn depends on the laser spatial profile at the mask and on the spatial filtering of light scattered from the edges of individual pixels on the mask. ${ }^{8}$ With a Gaussian spatial profile and with the assumption that spatial filtering subsequent to the mask eliminates all Hermite-Gaussian spatial modes, except for the fundamental Gaussian, the temporal envelope will also be Gaussian and hence symmetric. ${ }^{8}$ The observed asymmetry could arise from violation of either of these assumptions. Indeed, we observed that the degree of asymmetry was affected by adjusting the position and the aper-
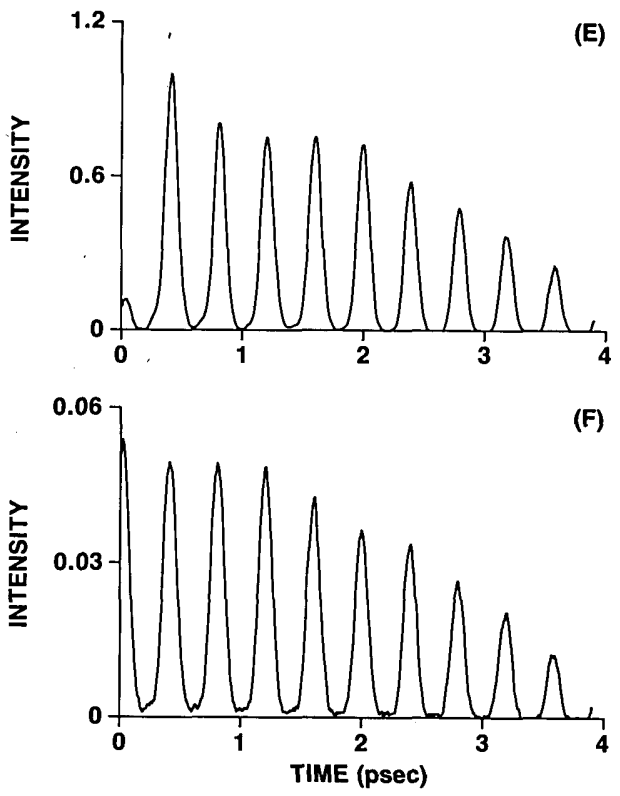

(F) 
quiring an amplitude mask that varies continuously with position. Furthermore, the combination of fast and slow features ( $<100$-fsec rise time, $>2$-psec pulse duration) necessitates patterning of the spectrum over a large dynamic range. Thus generation of the square pulse provides information about any symmetric pulse shape incorporating femtosecond features into a several-picosecond envelope.

The spectrum of a square pulse of duration $T$ is shaped as a sinc function, given by

$$
E(f)=E_{0} T \frac{\sin (\pi f T)}{\pi f T} .
$$

The corresponding mask is specified by

$$
M(x)=\frac{\sin \left(\pi x / x_{0}\right)}{\pi x / x_{0}}
$$

where $M(x)$ is the masking function, $x_{0}=(T \delta f / \delta x)^{-1}$, and $\delta f /$ $\delta x=2.63 \mathrm{THz} / \mathrm{mm}$ is the spatial dispersion. We implement the desired masking function [Eq. (3)] by using both an amplitude mask and a separate phase mask. The phase mask, which imparts the required alternating sign to the filter, is fabricated as before by reactive-ion etching of the appropriate regions of the mask. The variable-transmission amplitude mask consists of a series of opaque metal lines with widths and spacings that are varied in order to obtain the desired transmission. ${ }^{7}$ If the laser spot size is larger than the widths and spacings of the lines, the transmission must be averaged over the local metal pattern, taking diffraction effects into account. ${ }^{8}$ After the mask, the electricfield amplitude of the main undiffracted beam is given by

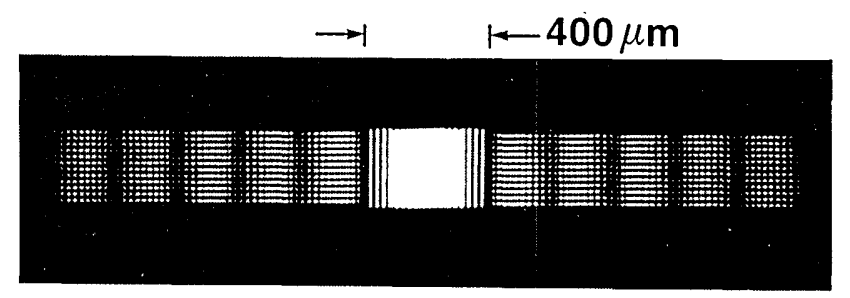

Fig. 7. Photograph of an amplitude mask used for generating a square pulse. Transparent regions are bright; opaque regions are dark.

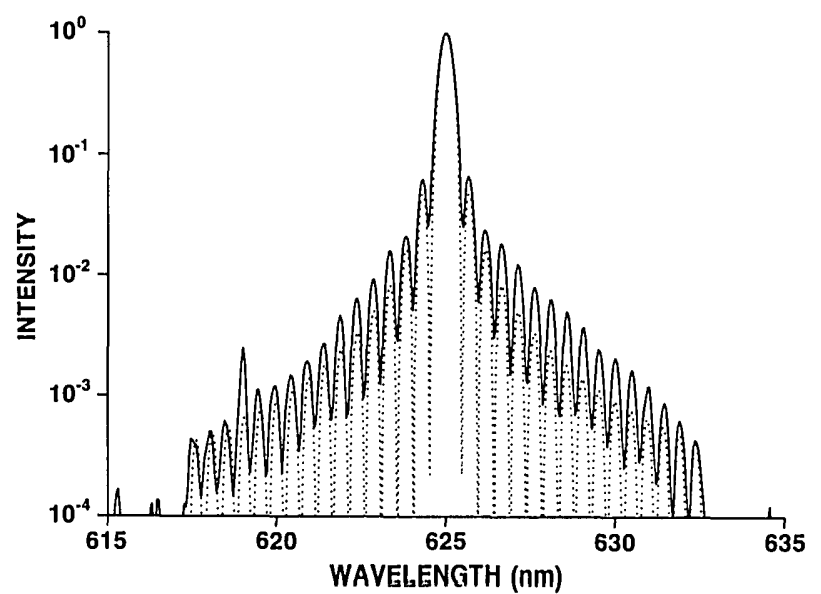

Fig. 8. Power spectra for a square pulse: solid trace, measured spectrum patterned by phase and amplitude masks; dotted trace, an actual sinc function (as a function of wavelength). the incident amplitude multiplied by the fraction of the mask area not covered by metal. The fraction of the intensity that is transmitted and not diffracted is the square of the fraction of the transparent area.

Figure 7 shows a transmission photograph of an amplitude mask. The bright parts of the photo are the transparent regions; the metal regions are dark. The sinc function is truncated after five sidelobes on each side of the central lobe; the width $x_{0}$ of each sidelobe is $200 \mu \mathrm{m}$, corresponding to 526 $\mathrm{GHz}$. The pattern includes one opaque line every $20 \mu \mathrm{m}$; the width of each line is varied in accordance with Eq. (3). Outside the central lobe $\left(|x|>x_{0}\right)$, a two-dimensional grid of lines is utilized in order to increase the available dynamic range. The minimum feature size (line or space) is $2 \mu \mathrm{m}$. The mask was prepared by evaporating a $2500-\AA$ titanium/ $500-\AA$ gold film onto a fused-silica substrate, followed by photolithographic patterning with a chemical etch to remove regions of unwanted metal. Before patterning was conducted the transmission through the metal film was below $10^{-4}$ (in terms of intensity). Leakage through thinner films with substantially higher transmission (e.g., $\simeq 1.4 \%$ for $300-\AA$ titanium $/ 200-\AA$ gold) leads to extraneous and undesirable features in the square-pulse data.

Figure 8 shows a semilog plot of a power spectrum produced over a $5.12-\mathrm{mm}$ window, with $x_{0}=160 \mu \mathrm{m}$ and with both amplitude and phase masks in place. Fifteen sidelobes are evident on each side of the central lobe, illustrating a dynamic range approaching $10^{4}: 1$, with an excellent signalto-noise ratio. The dotted trace is an actual sinc function (as a function of a wavelength). At the short-wavelength extreme of the spectrum, we note a slight $0.1-\mathrm{nm}$ shift between the data and the analytic sinc function. This shift arises because of higher-order spatial dispersion $\left(\mathrm{d}^{2} \lambda / \mathrm{d} x^{2}\right)$, which we have not accounted for in the mask design. Nevertheless, on the basis of the detailed control and the high dynamic range apparent in the spectrum, we would anticipate that square pulses would be generated with excellent fidelity.

A series of cross-correlation measurements of ultrafast square pulses is shown in Fig. 9. The pulse durations are 1, 2 , and 2.5 psec, corresponding to $x_{0}=400,200$, and $160 \mu \mathrm{m}$, respectively. For each $x_{0}$, two square pulses are shown, the first generated using a relatively narrow spectral window and the second generated using a wider window that accommodates essentially the entire laser bandwidth. The rise and fall times are found to be of the order of $100 \mathrm{fsec}$ (after accounting for the finite duration of the correlating pulse). The ripple present on those square pulses produced using the narrower windows arises because of the truncation of the spectrum; with the wider windows, the ripple nearly disappears. The ripple and the overshoot evident in the data are in good qualitative agreement with calculations. This agreement is illustrated by Fig. 10, which shows the theoretical intensity profile of the 2-psec square pulse shown in Fig. $9(C)$. This trace was obtained from the Fourier transform of a truncated sinc function, with $x_{0}=200 \mu \mathrm{m}$ and with five sidelobes per side. Square pulses with reduced ripple could be obtained by avoiding truncation of the spectrum and by instead using a more gentle spectral apodization.

Some discrepancies between data and theory do appear. For example, in the data of Fig. 9(C), the overshoot on the rising and falling edges is larger than expected, and the 

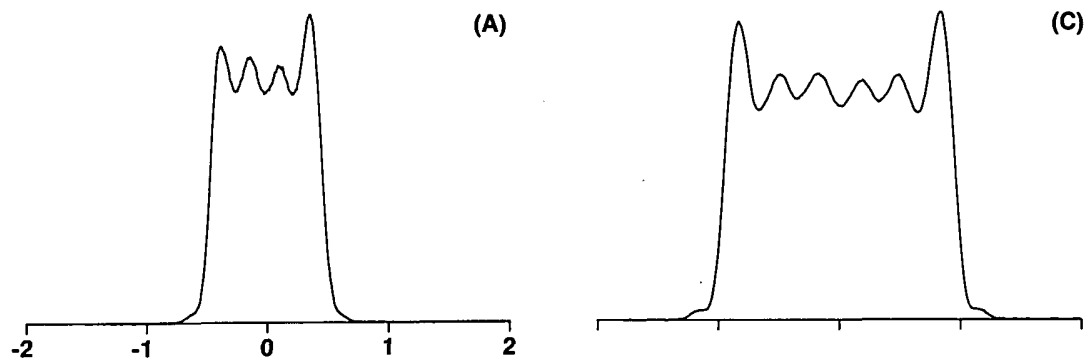

(C)
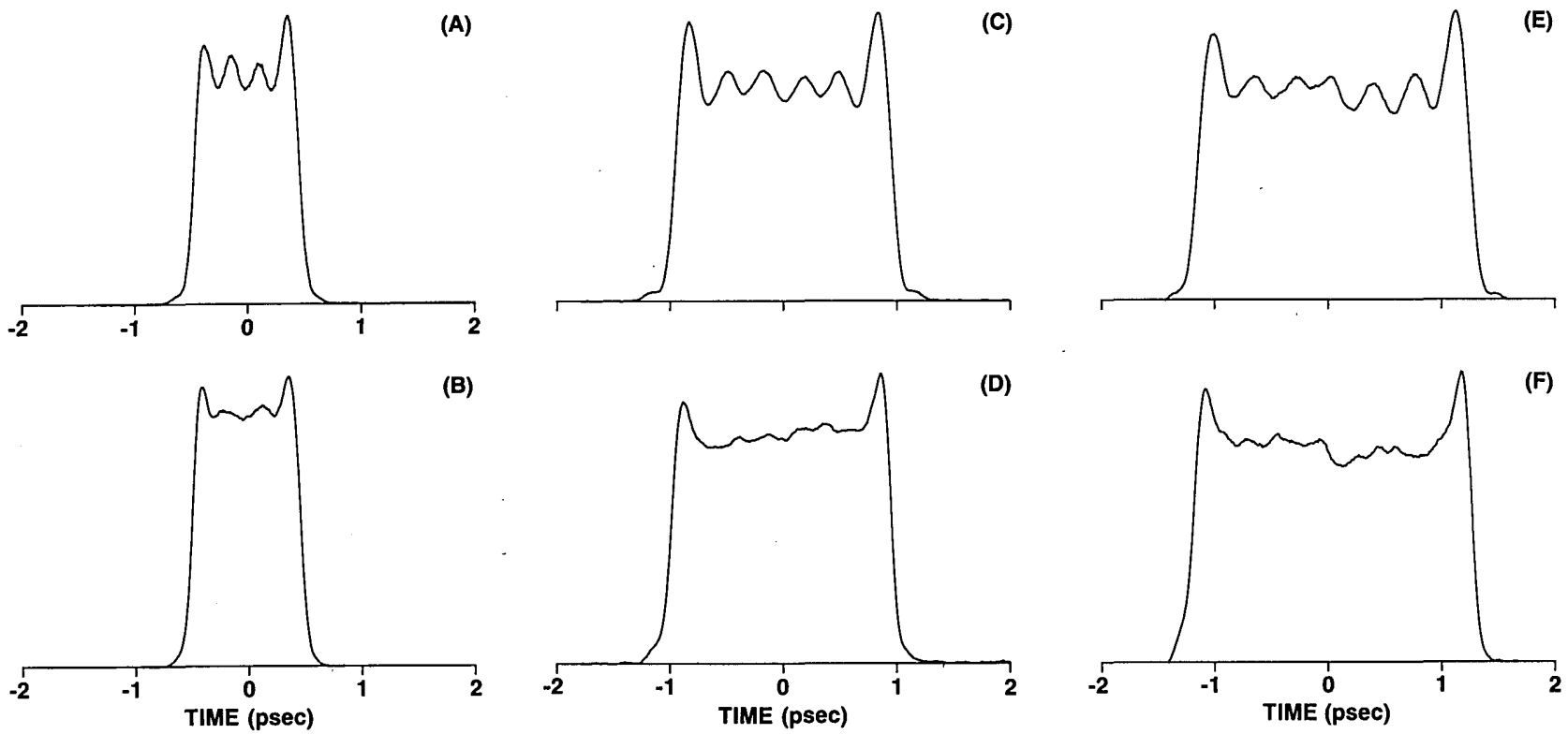

Fig. 9. Cross-correlation measurements of ultrafast square pulses: (A) pulse duration $T=1.0$ psec, sidelobe width $x_{0}=400 \mu \mathrm{m}$, number of sidelobes (per side) $N=3$; (B) $T=1.0 \mathrm{psec}, x_{0}=400 \mu \mathrm{m}, N=6$; (C) $T=2.0$ psec, $x_{0}=200 \mu \mathrm{m}, N=5$; (D) $T=2.0 \mathrm{psec}, x_{0}=200 \mu \mathrm{m}, N=12$; (E) $T=2.5 \mathrm{psec}, x_{0}=160 \mu \mathrm{m}, N=6$; (F) $T=2.5 \mathrm{psec}, x_{0}=160 \mu \mathrm{m}, N=15$.

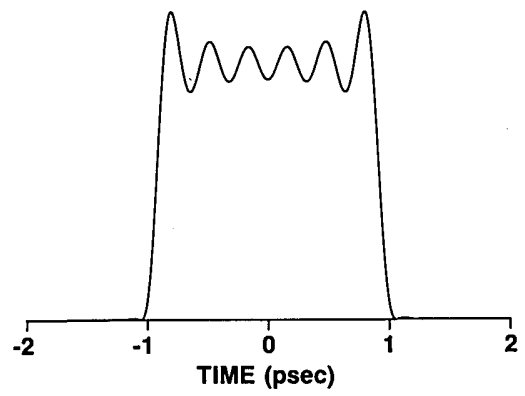

Fig. 10. Theoretical intensity profile of the 2-psec square pulse shown in Fig. $9(\mathrm{C})$.

ripple is not perfectly symmetric. Furthermore, many of the data exhibit a small shoulder in the region where the intensity is falling to zero. These differences may be attributed to a number of factors that are not included in the calculations. First, the laser spectrum is not flat, and the spectral phase is not completely uniform, especially for the wider spectral windows [Figs. 9(B), 9(D), and 9(F)]. Second, the transmission of the amplitude masks does not precisely reproduce the specified sinc function, and the phase masks typically deviate from the desired $\pi$ phase shift by $1-$ $2 \%$. Finally, as pointed out in reference to Fig. 8 , we have not corrected for the variation in $\delta f / \delta x$ as a function of wavelength (estimated at $\simeq 4.4 \%$ over a $10-\mathrm{nm}$ bandwidth).

\section{CONSTRAINTS}

Our pulse-shaping technique is based on linear filtering and is subject to various constraints common to any linear filtering technique. Here we discuss constraints that govern the complexity of the shaped waveform and the insertion loss of the spectral filter.

The first constraint arises because of finite bandwidth and spectral resolution. The shortest temporal feature that can be realized, $\delta t$, is inversely related to the total bandwidth $B$ $(B \delta t \simeq 0.44)$, and the maximum temporal window $T$ is inversely related to the finest achievable spectral feature $\delta f$ ( $\delta f T \simeq 0.44)$. The number of distinct spectral features that may be placed into the available bandwidth provides a useful measure $\eta$ of the complexity of the shaped pulse:

$$
\eta=B / \delta f
$$

Because $B / \delta f \simeq T / \delta t, \eta$ also describes the number of independent temporal features that may be synthesized into a waveform. This measure is related to the maximum timebandwidth product: $B T \simeq 0.44 \eta . .^{28}$

As discussed in Ref. 8, $\delta f$ is related to the spatial dispersion and to the spot size associated with any single frequency component. These quantities depend in turn on the angular dispersion of the grating and the divergence of the input beam. For gratings used at Littrow, $\eta$ is given by

$$
\eta=\frac{\Delta \lambda}{\lambda} \frac{\pi}{(\ln 2)^{1 / 2}} \frac{W}{d \cos \left(\theta_{d}\right)},
$$

where $\Delta \lambda$ is the bandwidth (in units of wavelength), $W$ is the $e^{-1}$ field radius of the input beam, $d$ is the grating period, and $\theta_{d}$ is the diffraction angle. ${ }^{29}$ With the current setup $(\lambda=$ $620 \mu \mathrm{m}, \Delta \lambda \simeq 10 \mathrm{~nm}, W \simeq 2 \mathrm{~mm}, 1 / d=1700 \mathrm{~mm}^{-1}$, and $\theta_{d} \simeq$ $\left.35^{\circ}\right), \eta$ is approximately 250 , corresponding to a 14-psec temporal window. If we assume a 3000 -line/mm grating and a 5 -mm beam radius, we obtain $\eta \simeq 2500$ ! Full utilization of such high $\eta$ values will require fabrication of masks to tight tolerances and correction for higher-order spatial-dispersion terms.

The insertion loss of the pulse shaper arises because of three separate mechanisms: (1) diffraction efficiency of the gratings, (2) scattering from the edges of individual pixels on the masks, and (3) attenuation imposed by amplitude masks. When a diffraction efficiency of $80 \%$ per grating is assumed, the first mechanism contributes a loss of $36 \%$ (2 
dB). Mechanism (2) becomes important only when the number of pixels approaches $\eta$ (i.e., when the pixel size becomes comparable with the spot size of the individual spectral components). For any particular mask, the energy lost to scattering may be computed by using the formulas in Ref. 8. As a concrete example, we consider a single repetition of a maximal length sequence of length $P$. The energy lost depends mainly on $\eta / P$ and not on $P$ itself. For $\eta / P=2$, we compute $\simeq 33 \%$ energy loss $(1.7 \mathrm{~dB})$ for code length $P=$ 63 or $P=127$; for $\eta / P=1$, we obtain $\simeq 57 \%$ loss $(3.7 \mathrm{~dB})$ for the same values of $P$.

Mechanism (3) plays a role only when amplitude masks are used. If $\delta t_{\text {in }}$ and $\delta t_{\text {out }}$ are the durations of the input pulse and the shaped waveform, respectively, then the amplitude mask transmits only $\delta t_{\text {in }} / \delta t_{\text {out }}$ (roughly) of the incident energy. Compared with the input intensity, the peak intensity of the shaped pulse is reduced by the factor $\left(\delta t_{\text {in }} / \delta t_{\text {out }}\right)^{2}$. If a phase-only mask is used, there is no energy loss, except for mechanisms (1) and (2); however, the peak intensity is reduced by $\delta t_{\text {in }} / \delta t_{\text {out }}$.

\section{SPECTRAL PHASE CODING OF FEMTOSECOND PULSES}

The ability to manipulate ultrashort pulses precisely will lead to important applications in high-speed communications. We previously proposed ${ }^{13}$ that encoding and decoding of coherent ultrashort pulses could form the basis for a code-division multiple-access (CDMA) optical telecommunications network, in which multiple access would be achieved by assigning different, minimally interfering code sequences to various subscriber pairs. ${ }^{1}$ In particular, we showed that coherent femtosecond pulses could be spread into picosecond-duration pseudonoise bursts by impressing pseudorandom phase codes onto the optical frequency spectrum, and we demonstrated that subsequent decoding of the spectral phases would restore the original pulse. Below we describe new results on encoding and decoding using $M$ sequence phase codes. As pointed out in Subsection 4.A, $M$ sequences are widely utilized in spread spectrum communications, and their properties are well known. ${ }^{1}$ Several of these properties are particularly well suited to ultrashort pulse CDMA.

A pseudorandom binary phase mask, patterned according to a single repetition of a length $127 \mathrm{M}$-sequence code is depicted in Fig. 11(A). The mask consists of a 2.5-mm (6.6$\mathrm{THz}$ ) clear aperture divided into 127 equal pixels, each of which contributes a phase shift of either 0 or $\pi$. Using $M$ sequences, we can generate a large set of minimally interfering code sequences. Any cyclic shift of the original $M$ sequence results in a new $M$ sequence. In this way, $P$ distinct codes may be obtained, where $P$ is the length of the sequence. Cross-correlation measurements of encoded waveforms are shown in Figs. 11(B) and 11(C) for two different shifts. As seen, spectral phase coding spreads incident 75fsec pulses into complicated pseudonoise bursts, filling 20 psec envelopes. Although the details of Figs. 11(B) and 11(C) are completely different, the duration and the complexity of these two waveforms are similar. Comparable results will be obtained by using any other cyclic shift of the mask. Previously we demonstrated spectral phase coding using a 44-element mask (not an $M$ sequence) and obtained a
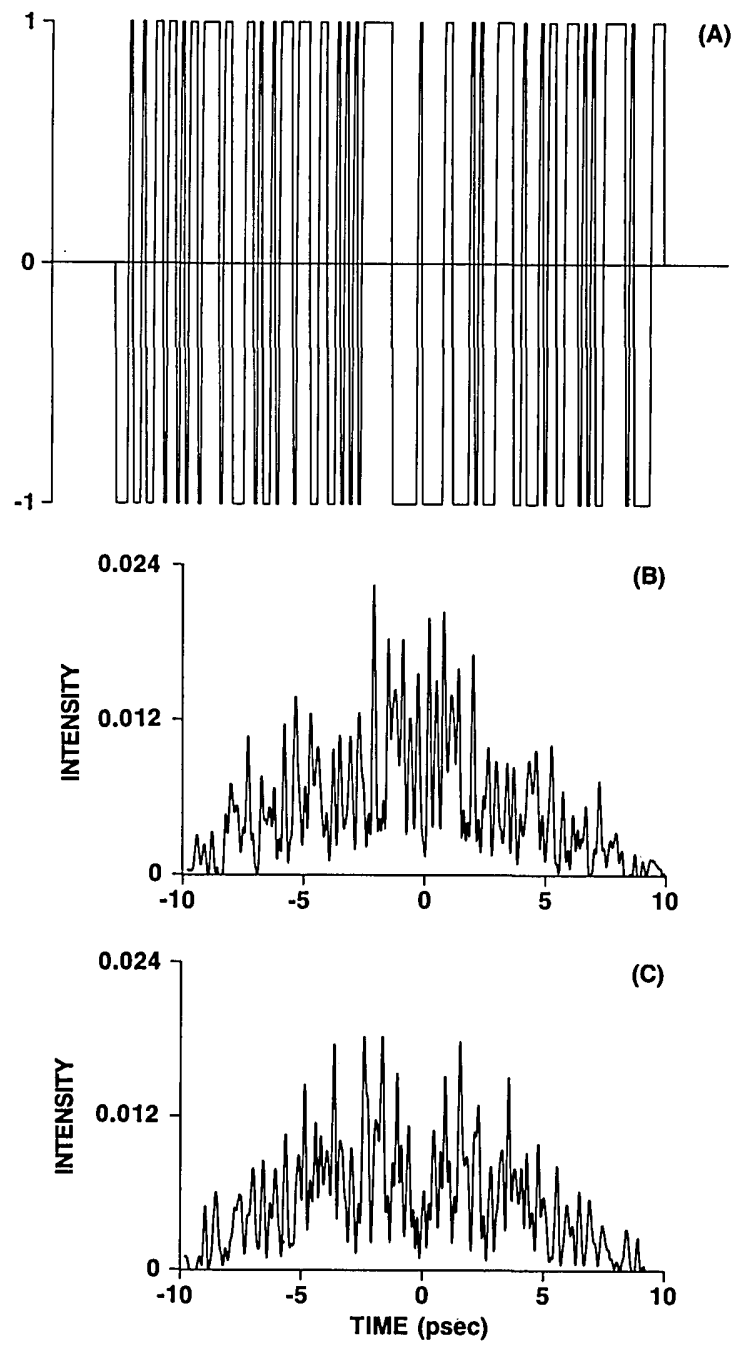

Fig. 11. Spectral phase coding. (A) Binary phase mask patterned according to a length $127 \mathrm{M}$ sequence. The pattern is $2.5 \mathrm{~mm}$ wide, and the vertical axis corresponds to transmission of +1 or -1 (phase shift of 0 or $\pi$ ). (B), (C) Cross-correlation measurements of pseudonoise bursts generated using different shifts of the above mask. For (B), the mask was cyclically shifted 63 pixels to the right; for (C), the shift was 10 pixels. The intensity scale for the cross correlations is referenced to the intensity of a similarly windowed uncoded pulse.

coded noise burst of $\simeq 7$ psec in duration. ${ }^{13}$ Our current data occupy a broader time window and exhibit greater complexity because of the larger number of pixels in the phase mask.

Pseudonoise bursts generated in this way can be decoded back into coherent ultrashort pulses by placing a second phase-conjugate mask adjacent to the first phase mask. The phase-conjugate mask undoes the phase scrambling imposed by the first mask, thus restoring the initial pulse. We have performed autocorrelation measurements that demonstrate that pseudonoise bursts, as shown above, can be transformed back into femtosecond pulses, with no broadening compared with uncoded pulses windowed to the same spectral width. These autocorrelations are similar to previously published data, ${ }^{13}$ which show the autocorrelation of a pulse encoded and decoded by using 44-element phase masks, and therefore are not presented here.

When the second mask does not match the first, the spec- 
tral phases are rearranged but not unscrambled. The resultant spectral phases are patterned according to the product of the two phase masks; when these masks correspond to two different shifts of a single $M$ sequence, the product corresponds to yet another shift of the same $M$ sequence! Thus a CDMA system using $M$-sequence phase masks will possess a useful closure property: the set of unsuccessfully decoded waveforms is identical to the set of encoded waveforms. This ensures that any unsuccessfully decoded pulse will have the same low-intensity pseudonoise behavior as the encoded pulses.

Autocorrelation measurements of two pseudonoise bursts that result from unsuccessful decoding are shown in Fig. 12. Both traces are plotted on a vertical scale ranging from zero to 0.008 , relative to the peak autocorrelation signal from a properly decoded pulse. This intensity reduction could be used in a CDMA system to discriminate between correctly and incorrectly addressd information. ${ }^{13,30}$ Both autocorrelation traces contain a coherence spike riding upon a broad pedestal, as appropriate for a noise burst. ${ }^{31}$ The observed contrast ratios are $\simeq 1.4: 1$, in agreement with numerical calculations and significantly lower than the 2:1 contrast ratio expected when the field is a Gaussian random variable. Thus these pseudonoise bursts produced by spectral phase coding with $M$ sequences are smoother and less peaked than Gaussian noise bursts obtained, for example, from imperfectly mode-locked lasers. ${ }^{31}$ We also note that the two autocorrelations plotted in Fig. 12 are nearly identical, although they correspond to pseudonoise bursts with completely different details. This observation points to the common statistical behavior of pseudonoise bursts encoded using $M$ sequences from the same family.

The discussion above is aimed at the case of single pseudonoise bursts, arriving one at a time. The performance of an

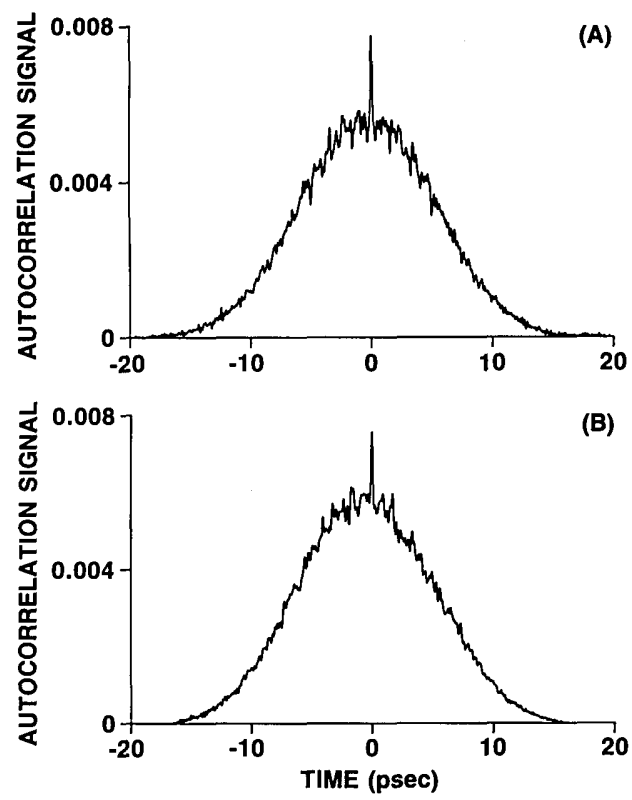

Fig. 12. Autocorrelation measurements of improperly decoded pseudonoise bursts. The encoding mask is shown in Fig. 11(A); the decoding masks are shifted versions of the phase conjugate of Fig. 11(A). (A) Shifted cyclically 63 pixels to the right. (B) Shifted 10 units to the right. The intensity scale for autocorrelations is referenced to the autocorrelation peak of a properly decoded pulse. actual CDMA system will depend on interference caused by a (variable) number of such pseudonoise bursts superimposed in time. The statistics of this interference will be analyzed elsewhere. ${ }^{32}$ The results included here indicate some of the tantalizing possibilities for new communications techniques based on frequency-domain manipulation of ultrashort light pulses.

\section{SUMMARY}

In this paper we have demonstrated the ability to manipulate femtosecond pulse shapes according to specification. Pulse shaping is achieved by spatially filtering optical frequency components spatially dispersed within a temporally nondispersive grating apparatus. Our apparatus permits realization of a wide and nearly arbitrary range of linear filters. We are able to pattern spectra over a high dynamic range $\left(\simeq 10^{-4}\right)$ and with good resolution. Resolutions in excess of 1000 should be attainable. We anticipate that real-time pulse shaping can be achieved by replacing the prefabricated masks used in the current work with highspeed multielement modulators. By utilizing a mask fabricated from a nonlinear material, our technique may be extended to perform nonlinear spectral filtering. Nonlinear filtering would provide the ability to perform pulse-shaping operations, such as time reversal, that are not possible with linear filtering.

The application of arbitrarily shaped pulses to communications and to spectroscopy will offer new opportunities and unprecedented flexibility in experimental design. In spectroscopy the ability to tailor the complex field amplitude and to synthesize special sequences of pulses will permit application of powerful coherent transient techniques on a subpicosecond time scale. In optical communications the application of parallel modulation in the frequency domain will translate into effective serial modulation rates in the terahertz range. As one example we discussed encoding and decoding of femtosecond pulses as the basis for ultrashort pulse CDMA. Other applications may include pulse-position modulation on a picosecond and subpicosecond time scale, dense wavelength-division multiplexing, and parallelto-serial conversion.

\section{ACKNOWLEDGMENTS}

We thank J. A. Salehi for many illuminating conversations regarding code-division multiple access and $\mathrm{D}$. E. Leaird for capable technical assistance. Stimulating discussions with O. E. Martinez and P. W. Smith are also gratefully acknowledged.

\section{REFERENCES AND NOTES}

1. R. Skaug and J. F. Hjelmstad, Spread Spectrum in Communications (Peregrinus, London, 1985).

2. J. Desbois, F. Gires, and P. Tournois, "A new approach to picosecond laser pulse analysis, shaping and coding," IEEE J. Quantum Electron. QE-9, 213 (1973).

3. J. Agostinelli, G. Harvey, T. Stone, and C. Gabel, "Optical pulse shaping with a grating pair," Appl. Opt. 18, 2500 (1979).

4. C. Froehly, B. Colombeau, and M. Vampouille, "Shaping and analysis of picosecond light pulses," in Progress in Optics $X X$ E. Wolf, ed. (North-Holland, Amsterdam, 1983), pp. 65-153. 
5. J. P. Heritage, R. N. Thurston, W. J. Tomlinson, A. M. Weiner, and R. H. Stolen, "Spectral windowing of frequency-modulated optical pulses in a grating compressor," Appl. Phys. Lett. 47, 87 (1985).

6. J. P. Heritage, A. M. Weiner, and R. N. Thurston, "Picosecond pulse shaping by spectral phase and amplitude manipulation," Opt. Lett. 10,609 (1985).

7. A. M. Weiner, J. P. Heritage, and R. N. Thurston, "Synthesis of phase coherent, picosecond optical square pulses," Opt. Lett. 11, 153 (1986).

8. R. N. Thurston, J. P. Heritage, A. M. Weiner, and W. J. Tomlinson, "Analysis of picosecond pulse shape synthesis by spectral masking in a grating pulse compressor," IEEE J. Quantum Electron. QE-22, 682 (1986).

9. M. Haner and W. S. Warren, "Generation of programmable, picosecond-resolution-shaped laser pulses by fiber-grating pulse compression," Opt. Lett. 12, 398 (1987).

10. R. L. Fork, B. I. Greene, and C. V. Shank, "Generation of optical pulses shorter than 0.1 psec by colliding pulse mode-locking," Appl. Phys. Lett. 38, 671 (1981).

11. J. A. Valdmanis, R. L. Fork, and J. P. Gordon, "Generation of optical pulses as short as 27 femtoseconds directly from a laser balancing self-phase modulation, group-velocity dispersion, saturable absorption, and saturable gain," Opt. Lett. 10, 131 (1985).

12. A. M. Weiner and J. P. Heritage, "Picosecond and femtosecond Fourier pulse shape synthesis," Rev. Phys. Appl. 22, 1619 (1987).

13. A. M. Weiner, J. P. Heritage, and J. A. Salehi, "Encoding and decoding of femtosecond pulses," Opt. Lett. 13, 300 (1988).

14. E. B. Treacy, "Optical pulse compression with diffracton gratings," IEEE J. Quantum Electron. QE-5, 454 (1969).

15. A. M. Johnson, R. H. Stolen, and W. M. Simpson, " $80 \times$ singlestage compression of frequency doubled Nd:yttrium aluminum garnet laser pulses," Appl. Phys. Lett. 44, 729 (1984).

16. O. E. Martinez, " 3000 times grating compressor with positive group velocity dispersion: application to fiber compensation in 1.3-1.6 $\mu \mathrm{m}$ region," IEEE J. Quantum Electron. QE-23, 59 (1987).

17. J. P. Heritage, A. M. Weiner, and O. E. Martinez, "Stabilized subpicosecond pulse compression due to multiple-order stimulated Raman scattering," J. Opt. Soc. Am A 4(13), P69 (1987).

18. M. Pessot, P. Maine, and G. Mourou, " 1000 times expansion/ compression of optical pulses for chirped pulse amplification," Opt. Commun. 62, 419 (1987)

19. K. Tai, A. Tomita, J. L. Jewell, and A. Hasegawa, "Generation of subpicosecond solitonlike optical pulses at $0.3 \mathrm{THz}$ repetition rate by induced modulational instability," Appl. Phys. Lett. 49, 236 (1986)

20. J. P. Heritage, A. M. Weiner, and R. N. Thurston, "Fourier transform picosecond pulse shaping and spectral phase measurement in a grating pulse-compressor," in Ultrafast Phenomena $V$, G. R. Fleming and A. E. Siegman, eds. (Springer-Verlag, Berlin, 1986), pp. 33-37.

21. J. E. Rothenberg, D. Grischkowsky, and A. C. Balant, "Observa- tion of the formation of the $0 \pi$ pulse," Phys. Rev. Lett. 53, 552 (1984).

22. A. Hasegawa and F. Tappert, "Transmission of stationary nonlinear optical pulses in dispersive dielectric fibers. II. Normal dispersion," Appl. Phys. Lett. 23, 171 (1973).

23. P. Emplit, J. P. Hamaide, F. Reynaud, C. Froehly, and A. Barthelemy, "Picosecond steps and dark pulses through nonlinear single mode fibers," Opt. Commun. 62, 374 (1987).

24. D. Krokel, N. J. Halas, G. Giuliani, and D. Grischkowsky, "Dark pulse propagation in optical fibers," Phys. Rev. Lett. 60, 29 (1988).

25. M. R. Schroeder, Number Theory in Science and Communication (Springer-Verlag, Berlin, 1986)

26. Y.-X. Yan, E. B. Gamble, and K. A. Nelson, "Impulsive stimulated scattering: general importance in femtosecond laser pulse interactions with matter, and spectroscopic applications," J. Chem. Phys. 83, 5391 (1985).

27. K. A. Nelson, Massachusetts Institute of Technology, Cambridge, Massachusetts 02139 (personal communication).

28. The relations $B \delta t \simeq 0.44$ and $\delta f T \simeq 0.44$ are derived assuming Gaussian line shapes for the power spectrum and for the finest achievable spectral feature, with $B, \delta f, T$, and $\delta t$ all referring to FWHM intensity widths. If, instead, the power spectrum is rectangular, then $B \delta t \simeq 0.886$, and we obtain $T / \delta t \simeq 0.5 B / \delta f=$ $0.5 \eta$.

29. In Ref. 8 a slightly different complexity measure $m$ was introduced, defined in terms of spatial factors, such as the laser spot size at the mask and the physical width of the spatially dispersed spectrum. The complexity measure used here, $\eta$, is defined in Eq. (4) in terms of spectral features. The relationship is $\eta=m /(\ln 2)^{1 / 2}$.

30. In the present setup, encoding and decoding masks are placed adjacent to each other; in a real CDMA system the two masks would be located apart from each other at separate pulse-shaping stations. As a result, the contrast between correctly and incorrectly addressed information will be somewhat different in a real system than in the present data. The difference arises because of scattering from the edges of individual pixels on the phase masks. In a real system, frequency components impinging upon the edges of pixels will be attenuated by scattering, and decoded pulses will have narrow holes in their frequency spectra. The primary effect of these holes is to diminish the intensity of decoded pulses. When an encoding and a matching decoding mask are adjacent, however, much of the scattering is eliminated. Under the present circumstances, with length 127 $M$ sequences and with a resolving power $\eta=250$, we calculate that the intensity of decoded pulses would be diminished by $\simeq 50 \%$ in a real system. The contrast in second-harmonic intensity generated by correctly and incorrectly decoded pulses would be $\simeq 67: 1$, as opposed to the ratio of $\simeq 130: 1$ evident in Fig. 12. For the case of high resolving power $(\eta / P \gg 1)$, the distinction discussed above disappears.

31. E. P. Ippen and C. V. Shank, "Techniques for measurement," in Ultrashort Light Pulses, S. L. Shapiro, ed. (Springer-Verlag, Berlin, 1977), pp. 85-88. 